\title{
Magnifying endoscopic observation of mantle cell lymphoma in the stomach using the narrow-band imaging system
}

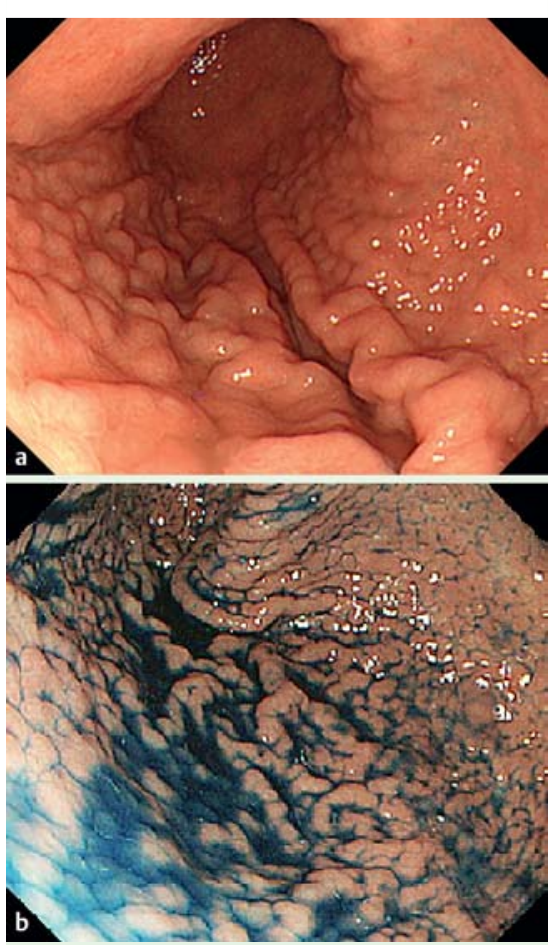

Fig. 1 a Conventional endoscopic image. Upper endoscopy revealed multiple protruding lesions in the stomach. $\mathbf{b}$ Endoscopic image of the same lesion after dye spraying (indigo carmine).

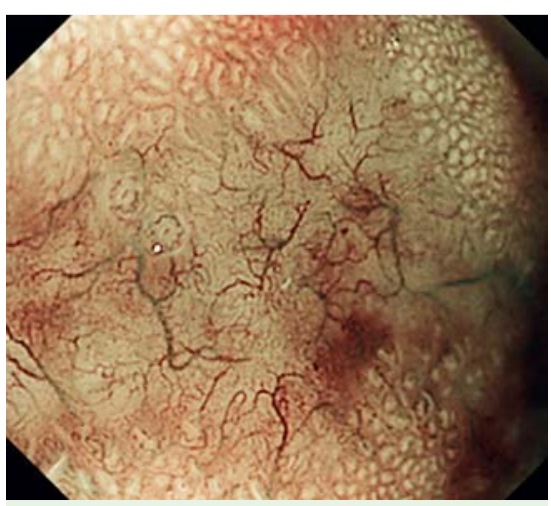

Fig. 2 Magnifying endoscopy combined with narrow-band imaging (NBI). The polypoid lesions show disappearance of the surface structure and a tree-like appearance of abnormal blood vessels.

Multiple lymphomatous polyposis (MLP) is characterized by multiple polypoid tumors affecting several segments of the gastrointestinal tract due to involvement in Hodgkin's or non-Hodgkin's lymphoma

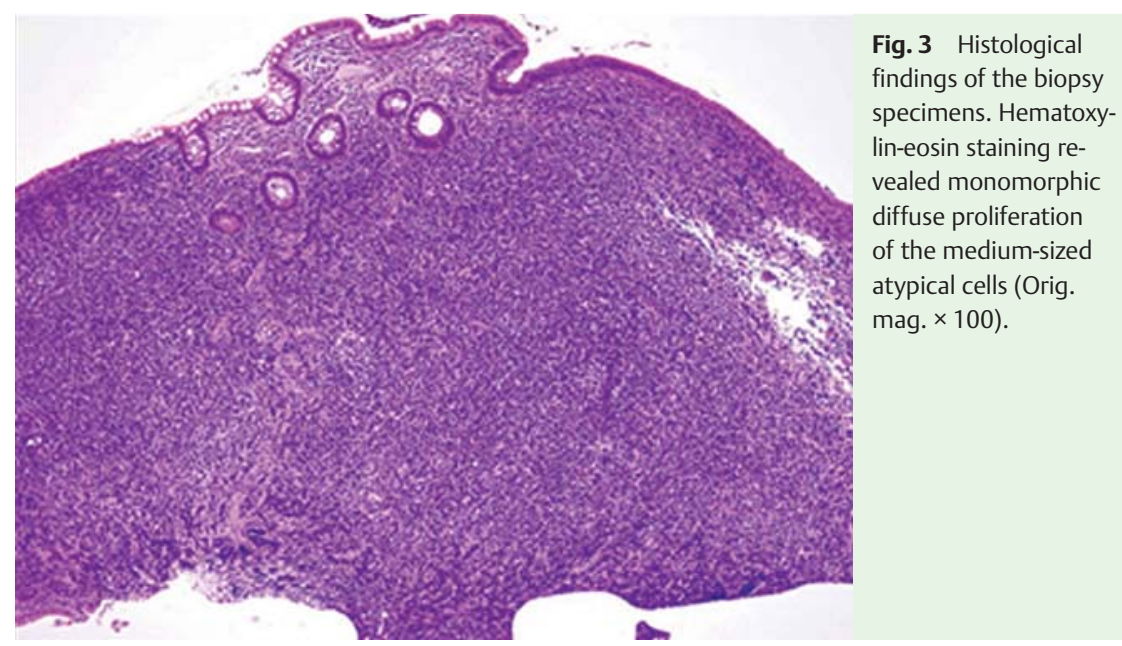

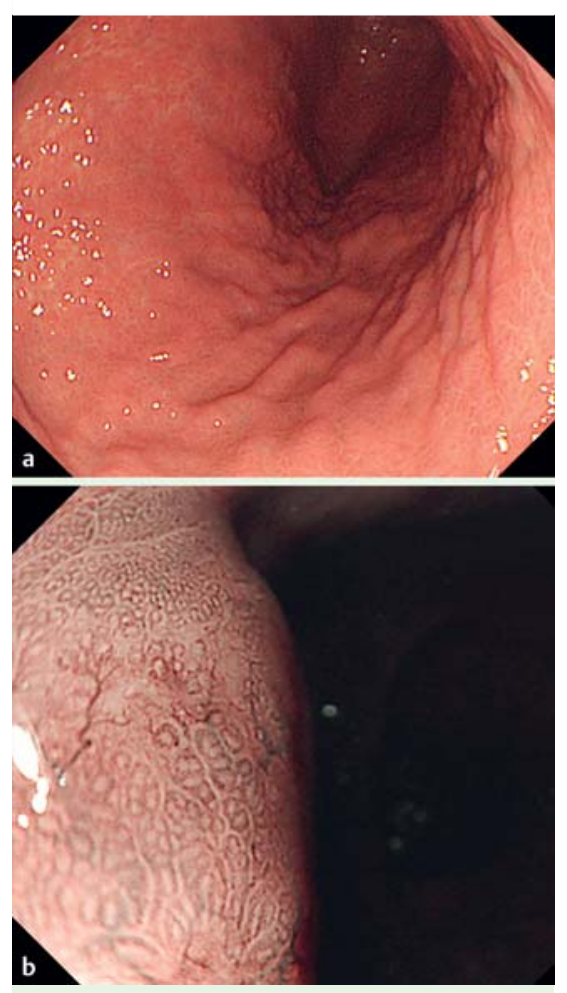

Fig. 4 a Conventional endoscopic image after chemotherapy with rituximab added to cyclophosphamide, doxorubicin, vincristine, and prednisone (R-CHOP). b Magnifying endoscopy with NBI shows remaining abnormal vessels resembling tree trunks with long, bare branches.

[1 - 3]. The histologic picture of MLP is in most cases consistent with mantle cell lymphoma, but some patients with mucosa-associated lymphoid tissue lymphoma and follicular lymphoma presenting with
MLP have recently been reported [4]. This is the first report of magnifying endoscopic observation of MLP using narrowband imaging (NBI).

A 74-year-old woman had epigastric pain. Upper gastrointestinal endoscopy revealed multiple protruding lesions in the stomach $(\bullet$ Fig. 1 a, b).

Magnifying endoscopy combined with NBI showed disappearance of the surface structure and the appearance of abnormal blood vessels looking like tree trunks with long, bare branches on the polypoid lesions ( $\bullet$ Fig. 2 ).

Target biopsy specimens of the site with the blood vessels revealed monomorphic diffuse proliferation of medium-sized atypical cells ( $\bullet$ Fig. 3 ).

Immunohistochemical analysis was positive for CD20, CD5, and cyclin D1, and negative for CD10, CD23, and CD43, consistent with mantle cell lymphoma. Positron emission tomography combined with computed tomography (PET-CT) showed extensive accumulation in the lymph nodes (maximum standardized uptake value: 6.1). Abnormal accumulation was also observed in the stomach and colon, but not in the small intestine. Colonoscopy revealed multiple flat plate colliculi in the colon. The woman was diagnosed as having stage IV mantle cell lymphoma.

She was treated with six courses of a chemotherapy regimen comprising rituximab added to cyclophosphamide, doxorubicin, vincristine, and prednisone (RCHOP regimen). In upper gastrointestinal 
endoscopic images 7 months after the treatment, the multiple protruding lesions were inconspicuous. On magnifying endoscopic images using NBI, however, the abnormal vessels remained, and target biopsy revealed residual mantle cell lymphoma ( Fig. 4).

The vascular abnormality associated with mantle cell lymphoma was easy to detect using magnifying endoscopy combined with NBI. Magnifying endoscopy combined with NBI may improve diagnostic performance in the diagnosis of mantle cell lymphoma.

Endoscopy_UCTN_Code_CCL_1AB_2AD_3AB
K. Nonaka ${ }^{1}$, K. Ishikawa ${ }^{1}$, S. Arai ${ }^{1}$, M. Shimizu ${ }^{2}$, T. Sakurai ${ }^{2}$, M. Nishimura ${ }^{1}$, M. Nakao ${ }^{1}$, Y. Sasaki ${ }^{3}$, H. Kita ${ }^{1}$

1 Department of Gastroenterology, Saitama Medical University International Medical Center, Saitama, Japan

2 Department of Pathology, Saitama Medical University International Medical Center, Saitama, Japan

3 Department of Gastroenterology, Faculty of Medicine, Kumamoto University, Kumamoto, Japan

\section{References}

1 Harris NL, Jaffe ES, Stein H et al. A revised European-American classification of lymphoid neoplasms: a proposal from the International Lymphoma Study Group. Blood 1994; 84: 1361 - 1392

2 Jaffe ES, Harris NL, Stein $H$ et al. Pathology and genetics of tumours of haematopoietic and lymphoid tissues. WHO classification of tumours. Lyon: IARC Press, 2001
3 Cornes JS. Multiple lymphomatous polyposis of the gastrointestinal tract. Cancer 1961; 14: $249-257$

4 Chim CS, Shek TWH, Liang R. Malignant lymphomatous polyposis in MALT lymphoma of colon. J Clin Oncol 2003; 21: 953-955

\section{Bibliography}

DOI $10.1055 / \mathrm{s}-0029-1243916$

Endoscopy 2010; 42: E94 - E95

(c) Georg Thieme Verlag KG Stuttgart · New York . ISSN 0013-726X

Corresponding author

\section{H. Kita, MD}

Department of Gastroenterology Saitama Medical University International Medical Center

1397-1 Yamane Hidakashi Saitama

350-1298 Japan

Fax: +81-4-29840054

hkita@saitama-med.ac.jp 